\title{
Painéis aglomerados fabricados com mistura de partículas de madeiras tropicais
}

\author{
Particleboards manufacture with the mixture of tropical \\ woods
}

\author{
Wilson Henrique Negrão \\ Sérgio Augusto Mello da Silva \\ André Luis Christoforo \\ Francisco Antonio Rocco Lahr
}

\section{Resumo}

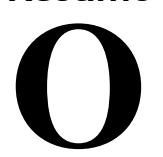

presente trabalho tem como objetivo investigar a influência do uso de três tipos de adesivo - resina poliuretana bicomponente derivada de mamona, ureia-formaldeído e Purbond - nas propriedades físicas (densidade aparente, teor de umidade, inchamento em espessura em 2 e em 24 h) e mecânicas (módulo de elasticidade e módulo de ruptura na flexão estática; resistência à tração perpendicular às faces) de painéis de partículas aglomeradas constituídos da mistura uniforme de quatro espécies de madeiras tropicais: cedrinho, cordia, fícus e paineira. Foi também avaliada a relação entre a densidade aparente e a resistência à tração perpendicular dos materiais fabricados. Com base em análises estatísticas, constatou-se que os melhores resultados das propriedades físicas foram obtidos com os materiais fabricados com o adesivo à base de mamona. Os materiais elaborados com resina ureia-formaldeído e Purbond apresentaram os melhores resultados para o módulo de elasticidade na flexão, e os fabricados apenas com ureia-formaldeído, os maiores valores do módulo de ruptura na flexão. Os compostos elaborados com a resina Purbond apresentaram os melhores resultados para a resistência à tração perpendicular, e, pelos resultados obtidos na análise de variância, constatou-se a validade da estimativa da resistência à tração pela densidade aparente dos painéis fabricados com o uso dos três tipos de adesivo.

Wilson Henrique Negrão Universidade Estadual Paulista Júlio de Mesquita Filho

Itha Solteira - SP - Brasil

Sérgio Augusto Mello da Silva Universidade Estadual Paulista Júlio de Mesquita Filho Ilha Solteira - SP - Brasil

André Luis Christoforo Universidade Federal de São Carlos São Carlos - SP - Brasil

Francisco Antonio Rocco Lahr Universidade de São Paulo São Carlos - SP - Brasil

Recebido em 20/04/13 Aceito em 23/06/14
Palavras-chave: Painéis de partículas. Madeiras tropicais. Resíduos.

\section{Abstract}

The aim of this study is to investigate the influence of the use of three types of adhesive - two-component polyurethane castor oil based resin, urea-formaldehyde and Purbond -in physical (density and moisture content, and thickness swelling at 2 and 24 hours), and mechanical properties (modulus of elasticity, and strength modulus in static bending, internal bond) in panels consisting of agglomerated particles of uniform mixture of four species of tropical woods: cedrinho, cordia, ficus and paineira. Moreover, the relationship between the bulk density and internal bond of the materials produced was also evaluated. Based on statistical analyses, the conclusion was made that the best results in terms of physical properties were obtained with the castor oil based adhesive. The materials produced with urea-formaldehyde and Purbond resin showed the best results for the modulus of elasticity in bending, while the ones produced with ureaformaldehyde had the best results regarding the strength modulus in bending. Moreover, compounds made from the Purbond resin had the best results for perpendicular tensile strength to faces. Based on the analysis of variance, the conclusion was made that it is valid to estimate of the tensile strength based on the density of the panels produced with any of the three types of adhesive.

Keywords: Particleboards. Tropical woods. Waste. 


\section{Introdução}

Os painéis derivados de madeira foram desenvolvidos como proposta de reaproveitamento dos resíduos gerados no processamento primário da madeira, de forma a explorar ao máximo o potencial dessa matéria-prima (TOMASELLI, 2000). As tecnologias para fabricação dos derivados de madeira tiveram grande impulso com o surgimento dos adesivos sintéticos, em especial aqueles à base de ureia-formaldeído (UF). Ainda hoje, em países como Brasil, a produção de painéis aglomerados é realizada empregando-se esse adesivo.

Devido ao crescimento do mercado moveleiro (MATTOS; GONÇALVES; CHAGAS, 2008), aumentou também a produção dos painéis aglomerados, motivando estudos de novas espécies para suprir a demanda da produção.

Segundo Maloney (1993), entre as espécies que apresentam potencial para a produção de painéis aglomerados podem ser citadas a Parkia giganto carpa ducke (faveira) e a Cordia goeldiana Huber (freijó). De acordo com Mendes et al. (2010), entretanto, qualquer material lignocelulósico pode, em princípio, ser utilizado para a confecção desses produtos.

Guimarães Júnior et al. (2012) propuseram a utilização de Cecropia hololeuca (embaúba) para a confecção de painéis aglomerados. Os resultados das propriedades físicas e mecânicas mostraram-se satisfatórios, e o produto final ficou dentro dos limites dos documentos normativos estabelecidos.

Mendes et al. (2012) confeccionaram painéis de espécies de rápido crescimento e, após testes físicos e mecânicos, concluíram que as espécies Pinus oocarpa e Cunningha mialanceolata possuem grande potencial para a produção de painéis aglomerados.

Estudo realizado por Blanchet, Cloutier e Riedl (2000) sobre a confecção de painéis de madeira aglomerada com resíduo de costaneiras de pínus possibilitou concluir ser viável o aproveitamento desta madeira e ser tecnicamente possível a utilização dos painéis de madeira aglomerada em recintos fechados.

Pesquisas sobre a produção de painéis de madeira aglomerada utilizando resíduos de costaneira de Eucalyptus saligna, Eucalyptus citriodora e Eucalyptus pilularis, realizadas por Iwakiri et al. (2001), avaliaram o comportamento dos painéis com misturas de resíduos e ureia-formaldeído. De acordo com os procedimentos normativos da CS 236-66, os painéis foram confeccionados com partículas a $3 \%$ de teor de umidade, $8 \%$ e $12 \%$ de teor de resina, densidade nominal de $0,8 \mathrm{~g} / \mathrm{cm}^{3} \mathrm{e}$ prensagem com $4 \mathrm{MPa}, 140{ }^{\circ} \mathrm{C}$ e $8 \mathrm{~min}$. As avaliações realizadas possibilitaram concluir que:

(a) os painéis com $12 \%$ de resina ureiaformaldeído apresentaram maior estabilidade dimensional, destacando-se os produzidos com Eucalyptus saligna;

(b) não ocorreram diferenças significativas entre o módulo de elasticidade para os diferentes níveis de resina $(8 \%$ e $12 \%)$;

(c) foram determinados melhores resultados de módulo de ruptura para os painéis com $12 \%$ de resina, confeccionados com partículas de Eucalyptus saligna, e somente para esta espécie a maior quantidade de resina (12\%) influenciou positivamente no módulo de ruptura;

(d) os resultados obtidos para a ligação interna dos painéis, em todos os tratamentos, foram superiores aos referenciados pelo código normativo CS 236-6; e

(e) os resíduos de costaneira, das espécies de eucalipto estudadas, podem ser recomendados para produção de painéis de madeira aglomerada.

Estudos no ciclo de prensagem em painéis de madeira aglomerada realizados por Albuquerque (2002), com o objetivo de avaliar a influência do incremento de água nas camadas superficiais do colchão de partículas e do incremento de resina e catalisador, possibilitaram concluir ser possível confeccionar painéis aglomerados com partículas finas, sem necessidade de se utilizar mais que $10 \%$ de ureia-formaldeído. Para o caso dos painéis com partículas grossas, devido ao aumento do teor de água nas camadas superficiais do colchão de partículas, observou-se que é necessário tempo superior a 4 min para conferir consolidação satisfatória.

Nascimento (2003) estudou a produção em laboratório de painéis de partículas homogêneas com madeira da região da Caatinga do Nordeste brasileiro: angico (Anedananthera macrocarpa), algaroba (Prosopis juliflora) e jurema-preta (Mimosa tenuiflora). Adotando a NBR 14810 (ABNT, 2006), os procedimentos experimentais foram definidos em função das propriedades físicas e mecânicas das espécies estudadas, das características do adesivo adotado e das partículas utilizadas para a confecção de painéis, considerando-se o emprego de partículas com 1,8 $\mathrm{mm}$ de comprimento, $10 \%$ de resina ureiaformaldeído com 5\% de solvente (base peso seco), tempo de prensagem de 10 min com temperatura entre 100 e $120{ }^{\circ} \mathrm{C}$, e pressão de prensagem de 4

104 Negrão, W. H.; Silva, S. A. M. da; Christoforo, A. L.; Lahr, F. A. R. 
MPa. Nessas condições, as propriedades físicas (densidade, absorção e inchamento) e mecânicas dos painéis foram satisfatórias, com valores médios de resistências (na flexão, adesão interna e arrancamento de parafuso) apresentando variabilidades equivalentes às dos painéis fabricados em escala industrial, de forma a concluir ser viável a fabricação de painéis de partículas homogêneas de boa qualidade com partículas de madeiras da Caatinga do Nordeste brasileiro.

A utilização de resíduo lignocelulósico na produção de painéis de madeira reconstituída, além de agregar valor, pode propiciar sustentabilidade, minimizar problemas de poluição ambiental e auxiliar no atendimento da demanda das indústrias de painéis aglomerados.

Segundo Mendes et al. (2010), o bagaço de cana é um exemplo de resíduo lignocelulósico que pode ser utilizado para a produção de painéis aglomerados, pois propicia a confecção de painéis com excelentes qualidades, considerando-se também que no Brasil o volume gerado é na ordem de 195 milhões de toneladas por ano (INSTITUTO..., 2010). Ainda de acordo com o mesmo autor, é possível a utilização de sabugo de milho, casca de arroz, caule de mandioca, casca de café e casca de amendoim, entre outros.

De acordo com Beraldo e José (2006), painéis aglomerados confeccionados com partículas de bambu e resina ureia-formaldeído apresentaram maiores valores de resistências mecânicas com relação aos painéis aglomerados confeccionados com partículas de pínus. Um aspecto importante sobre a produção de painéis aglomerados com partículas de bambu é a facilidade de cultivo dessa gramínea em praticamente todas as regiões brasileiras, com ciclos de colheitas curtos, o que propicia grande volume de matéria-prima para a produção dos painéis.

De acordo com Trianoski (2010), sob o ponto de vista tecnológico, a utilização de matérias-primas alternativas pode contribuir para melhorar a qualidade e as propriedades dos painéis de madeira aglomerada, principalmente devido às diferentes características inerentes a cada espécie. Pode ainda significar um incentivo à introdução dessas espécies em grandes áreas de florestas plantadas, uma vez assegurada a qualidade e os requisitos do produto em questão.

Campos et al. (2012) propuseram a confecção de painéis de madeira aglomerada misturando partículas de Eucalyptus grandis (eucalipto) e de Jatropha curcas (pinhão-manso), um subproduto da cadeia produtiva do biodiesel. $\mathrm{O}$ estudo mostrou que a proporção ideal de partículas do pinhão-manso na mistura é de 7,5\%, valor que não altera as propriedades físicas e mecânicas do painel. Embora a porcentagem seja pequena, o estudo mostra possíveis fins para subprodutos que geralmente são descartados sem nenhum critério.

Atualmente no Brasil, devido ao crescimento da geração de resíduos, evidencia-se o desenvolvimento de propostas que objetivam minimizar a poluição no meio ambiente. Entretanto, de modo geral, os resíduos são utilizados para produção de energia, e as indústrias brasileiras estão limitadas à utilização de tecnologia para processamento de madeira de pínus e eucalipto na produção de painéis aglomerados e painéis de fibra (SILVA et al., 2012).

Lima (2012) avaliou propriedades físicas e mecânicas de painéis fabricados com resíduos de madeiras da Amazônia (Dipterix polyphylla cumarurana; Brosimun paranarioides - amapá) e resina poliuretana à base de óleo de mamona, concluindo dos resultados obtidos ser viável a fabricação de painéis com o uso dos resíduos provenientes de ambas as espécies de madeira.

Com o intuito de investigar a viabilidade de produzir painéis aglomerados com a utilização de resíduos de madeira da Amazônia, este trabalho analisou as propriedades físicas e mecânicas de painéis constituídos da composição uniforme de quatro espécies de madeiras tropicais, com variação de três tipos de adesivos.

\section{Material e métodos}

Inicialmente foram realizadas coletas das espécies de madeiras mediante parceria com a Casa da Agricultura da cidade de Ilha Solteira (SP). As espécies coletadas, com suas respectivas densidades aparentes $\left(\rho_{\mathrm{ap}}\right)$, são apresentadas na Tabela 1.

As amostras coletadas foram seccionadas em pequenas toras, de aproximadamente $1 \mathrm{~m}$ de comprimento, com diâmetros variáveis. $\mathrm{Na}$ sequência, utilizando-se um micromoinho de facas (Figura 1a), foram produzidas partículas com comprimento de até $0,5 \mathrm{~cm}$ (Figura 1b).

Após serem processadas em moinho de facas, as partículas foram secas em estufa à temperatura de $60{ }^{\circ} \mathrm{C}$, até atingirem teor de umidade em torno de $5 \%$. Para confecção dos painéis foram utilizados os parâmetros de fabricação apresentados na Tabela 1, obtidos através de testes preliminares (flexão), empregando-se separadamente as resinas poliuretana bicomponente derivada de óleo de mamona, ureia-formaldeído e Purbond HB SB109. A resina ureia-formaldeído foi preparada com a 
inclusão de 5\% de solvente (NASCIMENTO, 2003), e a PU-mamona, com uma parte de poliol para uma de pré-polímero (SILVA e LAHR, 2008), ambas em função da massa seca das partículas.

Após as partículas atingirem o teor de umidade desejado, iniciou-se o processo para preparação do colchão de partículas (Figura 2a), adicionando-se resina e realizando-se pré-prensagem com pressão de compactação de $0,1 \mathrm{MPa}$. Esse procedimento propicia uma aproximação inicial entre as partículas e a consequente formação do colchão de partículas. Posteriormente à formação do colchão, os painéis foram fabricados (Figura 2b), de acordo com o ciclo de prensagem apresentado na Tabela 2.

Para fabricação dos painéis, de dimensões $400 \times 400 \times 10 \mathrm{~mm}$, fixou-se a massa de partículas em torno $1.280 \mathrm{~g}$, sendo utilizados $320 \mathrm{~g}$ de partículas por espécie de madeira em sua formação.

As propriedades físicas e mecânicas avaliadas foram: densidade aparente $\left(\rho_{\mathrm{ap}}\right)$, absorção de água em $2 \mathrm{~h}$ e em $24 \mathrm{~h}$ (Abs. $2 \mathrm{~h}$; Abs. $24 \mathrm{~h}$ ), inchamento em espessura em $2 \mathrm{~h}$ e em $24 \mathrm{~h}$ (Inc. 2 h; Inc. $24 \mathrm{~h}$ ), módulo de elasticidade na flexão (MOE), módulo de resistência na flexão (MOR) e resistência à tração perpendicular (RTP). Com exceção do módulo de elasticidade, obtido com os métodos e premissas de cálculo do código normativo ASTM D-1037 (AMERICAN..., 2006), as demais propriedades foram obtidas com o uso da norma brasileira ABNT NBR 14810 (ABNT, 2006). Além da norma brasileira, foram utilizadas para verificação de requisitos as normas CS236-66 (COMMERCIAL..., 1968), ANSI A208.1 (AMERICAN..., 1999) e EN 312 (EUROPEAN..., 2003). A Tabela 3 apresenta os valores-limite estabelecidos por esses documentos normativos como forma de verificar e avaliar a potencialidade dos materiais fabricados.

Para cada tipo de adesivo foram fabricados três painéis, sendo deles extraídos 20 corpos de prova para os ensaios de flexão $(250 \times 50 \times 10 \mathrm{~mm}), 12$ para os ensaios de adesão interna $(50 \times 50 \times 10 \mathrm{~mm})$ e outros 12 corpos de prova para a obtenção das propriedades físicas $(50 \times 50 \times 10 \mathrm{~mm})$.

Tabela 1 - Espécies de madeira coletadas

\begin{tabular}{ccc}
\hline Nome popular & Nome científico & $\boldsymbol{\rho}_{\mathbf{a p}}\left(\mathbf{g} / \mathbf{c m}^{\mathbf{3}}\right)$ \\
\hline Cedrinho & Cedrela odorata & 0,55 \\
Córdia & Cordia goeldiana & 0,50 \\
Figueira & Ficus insipida & 0,45 \\
Paineira & Ceiba pentandra & 0,35 \\
\hline
\end{tabular}

Figura 1 - Moinho de faca para produção de partículas com até $0,5 \mathrm{~cm}$ de comprimento

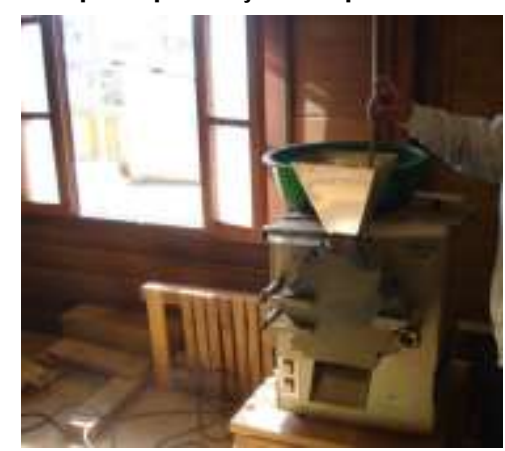

(a)

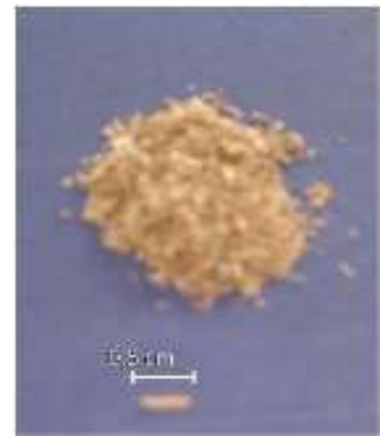

(b)

Tabela 2 - Parâmetros experimentais para confecção dos painéis aglomerados

\begin{tabular}{|c|c|c|c|c|}
\hline $\begin{array}{l}\text { Densidade nominal } \\
\qquad\left(\mathrm{g} / \mathrm{cm}^{3}\right)\end{array}$ & $\begin{array}{l}\text { Teor de } \\
\text { resina }(\%)\end{array}$ & $\begin{array}{l}\text { Ciclo de prensagem (min. } \\
\text { e seg.) }\end{array}$ & $\begin{array}{l}\text { Temperatura de } \\
\text { prensagem }\left({ }^{\circ} \mathrm{C}\right)\end{array}$ & $\begin{array}{c}\text { Pressão de } \\
\text { prensagem } \\
(\mathrm{MPa}) \\
\end{array}$ \\
\hline 0,80 & 10 & $\begin{array}{c}\text { 3' (prensagem inicial) } \\
\text { 30'” (alívio de pressão) } \\
\text { 7' (prensagem final) }\end{array}$ & 100 & 5 \\
\hline
\end{tabular}

106 Negrão, W. H.; Silva, S. A. M. da; Christoforo, A. L.; Lahr, F. A. R. 
Figura 2 - Formação do colchão de partículas (a) e do painel (b)

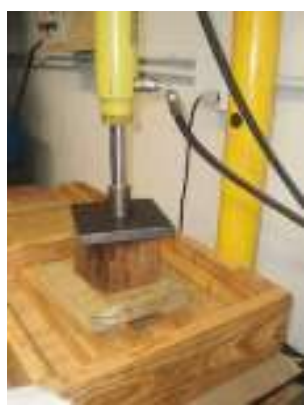

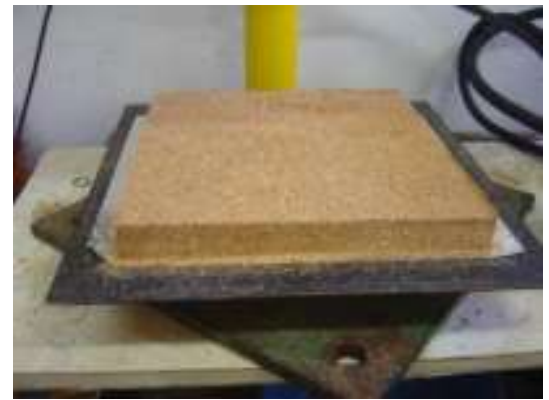

(a)

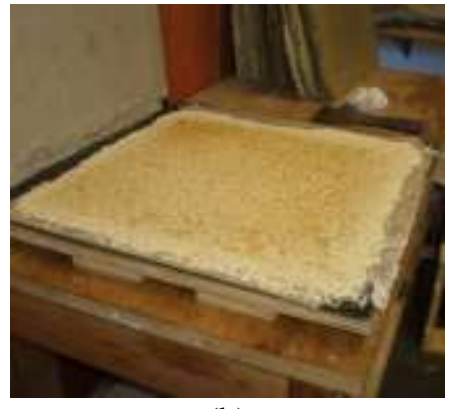

(b)

Tabela 3 - Valores de propriedades físicas e mecânicas de referência pelos códigos normativos utilizados

\begin{tabular}{cccccccc}
\hline Normas & $\begin{array}{c}\text { Espessura } \\
(\mathbf{m m})\end{array}$ & $\begin{array}{c}\boldsymbol{\rho} \\
\left(\mathbf{g} / \mathbf{c m}^{3}\right)\end{array}$ & $\begin{array}{c}\text { Inc. } \\
(\boldsymbol{\%})\end{array}$ & TU (\%) & $\begin{array}{c}\text { MOE } \\
(\mathbf{M P a})\end{array}$ & $\begin{array}{c}\text { MOR } \\
(\mathbf{M P a})\end{array}$ & $\begin{array}{c}\text { RTP } \\
(\mathbf{M P a})\end{array}$ \\
\hline CS236-66 (COMERCIAL..., 1968) & - & $>0,8$ & 55 & - & 2450 & 16,8 & 1,4 \\
ANSI A208.1 (AMERICAN..., & - & $>0,8$ & 8 & - & 2400 & 16,5 & 0,9 \\
1999) & $>6$ a 13 & - & 16 & - & 2300 & 16 & 0,4 \\
EN 312 (EUROPEAN..., 2003) & 8 a 13 & - & 8 & 5 a 11 & - & 18 & 0,4 \\
NBR 14810-2 (ABNT, 2006) & &
\end{tabular}

Os resultados das propriedades físicas e mecânicas foram avaliados com o uso da análise de variância (ANOVA) de Kruskal-Wallis (não paramétrica) e do teste de comparações múltiplas entre postos de Student-Newman-Keuls. Além da caracterização, foi avaliada a relação entre a densidade aparente $\mathrm{e}$ a resistência à tração perpendicular dos painéis, por tipo de adesivo, com o uso de teste de correlação e do modelo de mínimos quadrados para a regressão linear, de forma a estimar a resistência com o conhecimento da densidade.

\section{Resultados e discussões}

A Tabela 4 apresenta os resultados obtidos das propriedades físicas e mecânicas dos painéis para cada um dos três tratamentos utilizados, sendo $\bar{x}$ e $c v$ a média amostral e coeficiente de variação respectivamente.

O teor de umidade de ambos os painéis de produtos fabricados foi inferior ao valor de referência-limite estipulado pelo documento normativo brasileiro NBR 14810-2 (ABNT, 2006).

As densidades das chapas confeccionadas com ambos os tipos de adesivos foram próximas a $0,70 \mathrm{~g} / \mathrm{cm}^{3}$.

Os materiais fabricados com o uso da resina PUmamona apresentaram os menores valores para a absorção em 2 h e em 24 h, sendo $88 \%$ e $90 \%$ inferiores aos valores da absorção de água em $2 \mathrm{~h}$ dos painéis fabricados com ureia-formaldeído e Purbond respectivamente, e $71 \%$ e $76 \%$ inferiores aos valores da absorção de água em $24 \mathrm{~h}$ dos materiais elaborados com ureia-formaldeído e Purbond.

Assim como na absorção de água, os compostos elaborados com resina PU-mamona apresentaram os melhores resultados para o inchamento em espessura. $\mathrm{O}$ inchamento em espessura ( $2 \mathrm{~h}$ e $24 \mathrm{~h}$ ) dos painéis fabricados com as resinas PU-mamona e ureia-formaldeído mostraram ser inferiores ao limite estabelecido pela norma EN 312 (EUROPEAN..., 2003), o mesmo não ocorrendo com os materiais fabricados com o adesivo Purbond. Entretanto, apresentaram valores de inchamento inferiores ao limite estipulado pela norma CS236-66 (COMMERCIAL..., 1968) (Inc. $24 \mathrm{~h}$ ), definido para densidades superiores a 0,80 $\mathrm{g} / \mathrm{cm}^{3}$.

O módulo de resistência na flexão dos painéis fabricados com ambos os adesivos foi superior aos valores estipulados pelos quatro documentos normativos de referência utilizados, apresentando os maiores valores os compostos fabricados com adesivo ureia-formaldeído, sendo $42 \%$ superior em média ao MOR dos materiais confeccionados com os demais adesivos.

Os produtos fabricados com o adesivo Purbond apresentaram os maiores valores do módulo de elasticidade na flexão, sendo $36 \%$ e $184 \%$ superiores ao MOE dos materiais fabricados com ureia-formaldeído e PU-mamona respectivamente. Com relação aos limites estabelecidos pelas normas internacionais para módulo de elasticidade 
na flexão, apenas os materiais fabricados com o adesivo PU-mamona apresentaram valores inferiores, de $31 \%$ em relação ao estabelecido pela norma EN 312 (EUROPEAN..., 2003).

Os maiores valores da resistência à tração perpendicular foram provenientes dos painéis fabricados com o adesivo PU-mamona, sendo $39 \%$ e $120 \%$ superiores à RTP dos materiais fabricados com ureia-formaldeído e com Purbond respectivamente. Por se tratar de painéis com 10 mm de espessura, os valores da RTP de ambos os materiais fabricados atenderam satisfatoriamente aos limites de resistência das normas NBR 148102 (ABNT, 2006) e EN 312 (EUROPEAN..., 2003). Os compostos fabricados com PU-mamona apresentaram RTP superior ao limite da norma ANSI A208.1 (AMERICAN..., 1999), mas inferior ao valor de referência da norma CS236-66 (COMMERCIAL..., 1968).

Cabe ressaltar que os coeficientes de variação dos valores dos módulos de resistência e de elasticidade obtidos nos ensaios de flexão estática e dos módulos de resistência obtidos nos ensaios de resistência à tração perpendicular apresentaram sistematicamente valores inferiores a $20 \%$ para o coeficiente de variação. Este valor é aceito pelos fabricantes de painéis e também é objeto de recomendação por parte de Nascimento (2003) e Dias et al. (2005).

A Tabela 5 apresenta os resultados da análise de variância segundo o teste de Kruskal-Wallis e de comparações múltiplas entre postos de StudentNewman-Keuls para as variáveis-resposta investigadas, estando sublinhados os P-valores inferiores a 0,05 , considerados significativos a um nível de confiabilidade de $95 \%$. Os valores 1,2 e 3 dos grupos apresentados na Tabela 5 referem-se aos materiais fabricados com os adesivos PUmamona, ureia-formaldeído e Purbond respectivamente.

A Tabela 6 apresenta os resultados dos agrupamentos baseados nos dados da Tabela 5 . Letras diferentes determinam médias estatisticamente diferentes entre si, ao nível de $95 \%$ de probabilidade.

Os coeficientes de correlação de Pearson $(\rho)$ (densidade e resistência à tração perpendicular) e os respectivos P-valores dos testes por tipo de adesivo são apresentados na Tabela 7 .

Para o teste de correlação, a hipótese nula $\left(\mathrm{H}_{0}\right)$ estipulada consistiu na não existência de correlação $(\rho=0)$, implicando existência de correlação $(\rho \neq 0)$ para a hipótese alternativa $\left(H_{1}\right)$, a um nível de significância de 5\%. Pelos P-valores encontrados, foi constatada correlação entre a densidade aparente e a resistência à tração perpendicular para os três tipos de resinas utilizados (P-valor<0,005). Os gráficos de regressão linear e os resultados das estatísticas de regressão e da ANOVA são apresentados na Figura 3 e na Tabela 8 respectivamente.

Observa-se da Tabela 8 que o valor do $\mathrm{F}$ de significação para os três tipos de adesivo se apresentou inferior ao valor $\mathrm{F}$ do nível de significância; consequentemente, considerando-se que a análise foi realizada para um nível de significância 5\%, as regressões encontradas devem ser aceitas.

Tabela 4 - Resultados das propriedades físicas e mecânicas dos painéis fabricados

\begin{tabular}{ccccccc}
\hline & \multicolumn{2}{c}{ PU-mamona } & \multicolumn{2}{c}{ Ureia-formaldeído } & \multicolumn{2}{c}{ Purbond } \\
\hline Resposta & $\overline{\boldsymbol{x}}$ & $\boldsymbol{c} \boldsymbol{v}(\boldsymbol{\%})$ & $\overline{\boldsymbol{x}}$ & $\boldsymbol{c v}(\boldsymbol{\%})$ & $\overline{\boldsymbol{x}}$ & $\boldsymbol{c v}(\boldsymbol{\%})$ \\
\hline TU (\%) & 6,56 & 5 & 7,61 & 1 & 7,45 & 1 \\
$\rho_{a p}\left(\mathrm{~g} / \mathrm{cm}^{3}\right)$ & 0,73 & 8 & 0,65 & 10 & 0,61 & 8 \\
Abs. 2 h $(\%)$ & 6,44 & 15 & 54,81 & 10 & 62,54 & 14 \\
Abs. 24 h (\%) & 20,07 & 11 & 69,46 & 8 & 84,88 & 11 \\
Inc. 2 h (\%) & 3,46 & 13 & 12,94 & 8 & 31,33 & 23 \\
Inc. 24 h (\%) & 9,35 & 8 & 15,56 & 8 & 38,40 & 15 \\
MOR (MPa) & 19 & 13 & 27 & 14 & 19 & 11 \\
MOE (MPa) & 1581 & 17 & 3307 & 13 & 4500 & 13 \\
RTP (MPa) & 1,17 & 18 & 0,84 & 13 & 0,53 & 17 \\
\hline
\end{tabular}

108 Negrão, W. H.; Silva, S. A. M. da; Christoforo, A. L.; Lahr, F. A. R. 
Tabela 5 - Resultados da ANOVA e das comparações por Student-Newman-Keuls

\begin{tabular}{|c|c|c|c|c|c|c|c|c|c|}
\hline \multirow[b]{2}{*}{ Resposta } & \multirow[b]{2}{*}{$\mathbf{H}$} & \multirow[b]{2}{*}{ GL } & \multirow[b]{2}{*}{ P-valor } & \multicolumn{3}{|c|}{ Posto Médio } & \multicolumn{3}{|c|}{$\begin{array}{c}\text { Comparações Student-Newman- } \\
\text { Keuls (P-valor) }\end{array}$} \\
\hline & & & & $\mathbf{R 1}$ & $\mathbf{R 2}$ & $\mathbf{R 3}$ & $\begin{array}{l}\text { Grupos } \\
(1 \text { e } 2)\end{array}$ & $\begin{array}{l}\text { Grupos } \\
(1 \text { e 3) }\end{array}$ & $\begin{array}{l}\text { Grupos } \\
(2 \text { e 3) }\end{array}$ \\
\hline$T U$ & 35,5 & 2 & $\underline{0,0000}$ & 10,50 & 37,38 & 27,63 & 0,0001 & 0,0003 & 0,0630 \\
\hline$\rho_{a p}$ & 23,30 & 2 & $\underline{0,0000}$ & 32,33 & 17,79 & 10,83 & 0,0019 & 0,0001 & 0,1845 \\
\hline Abs. $2 h$ & 32,09 & 2 & $\overline{0,0000}$ & 10,50 & 31,75 & 33,25 & 0,0001 & 0,0001 & 0,7748 \\
\hline Abs. $24 \mathrm{~h}$ & 35,77 & 2 & $\underline{0,0000}$ & 10,50 & 27,42 & 37,58 & 0,0003 & 0,0001 & 0,0575 \\
\hline Inc. $2 h$ & 36,81 & 2 & $\underline{0,0000}$ & 10,50 & 26,75 & 38,25 & 0,0005 & 0,0001 & 0,0283 \\
\hline Inc. $24 \mathrm{~h}$ & 37,24 & 2 & $\overline{0,0000}$ & 10,50 & 26,50 & 38,50 & 0,0006 & 0,0001 & 0,0221 \\
\hline MOR & 12,68 & 2 & $\underline{0,0018}$ & 18,25 & 33,75 & 18,33 & 0,0010 & 0,9858 & 0,0033 \\
\hline$M O E$ & 32,76 & 2 & $\underline{0,0000}$ & 10,95 & 27,50 & 36,75 & 0,0004 & 0,0001 & 0,0777 \\
\hline$R T P$ & 34,58 & 2 & $\underline{0,0000}$ & 33,96 & 18,96 & 6,92 & 0,0014 & 0,0001 & 0,0217 \\
\hline
\end{tabular}

Tabela 6 - Agrupamentos do fator tipo de adesivo por resposta

\begin{tabular}{cccc}
\hline Resposta & PU-mamona & Ureia-formaldeído & Purbond \\
\hline$T U$ & $\mathrm{~B}$ & $\mathrm{~A}$ & $\mathrm{~A}$ \\
$\rho_{a p}$ & $\mathrm{~A}$ & $\mathrm{~B}$ & $\mathrm{~B}$ \\
$A b s .2 h$ & $\mathrm{~B}$ & $\mathrm{~A}$ & $\mathrm{~A}$ \\
$A b s .24 h$ & $\mathrm{~B}$ & $\mathrm{~A}$ & $\mathrm{~A}$ \\
$I n c .2 h$ & $\mathrm{C}$ & $\mathrm{B}$ & $\mathrm{A}$ \\
$I n c .24 h$ & $\mathrm{C}$ & $\mathrm{B}$ & $\mathrm{A}$ \\
$M O R$ & $\mathrm{~B}$ & $\mathrm{~A}$ & $\mathrm{~B}$ \\
$M O E$ & $\mathrm{~B}$ & $\mathrm{~A}$ & $\mathrm{~A}$ \\
$R T P$ & $\mathrm{~A}$ & $\mathrm{~B}$ & $\mathrm{C}$ \\
\hline
\end{tabular}

Tabela 7 - Resultados do teste de correlação

\begin{tabular}{ccc}
\hline Adesivo & $\boldsymbol{\rho}$ & P-valor \\
\hline PU-mamona & 0,809 & 0,019 \\
Ureia-formaldeído & 0,669 & 0,027 \\
Purbond & 0,793 & 0,012 \\
\hline
\end{tabular}

Figura 3 - Regressão linear entre a $\rho_{\mathrm{ap}}$ e a RTP dos painéis fabricados com os adesivos (a) PU-mamona, (b) ureia-formaldeído e (c) Purbond

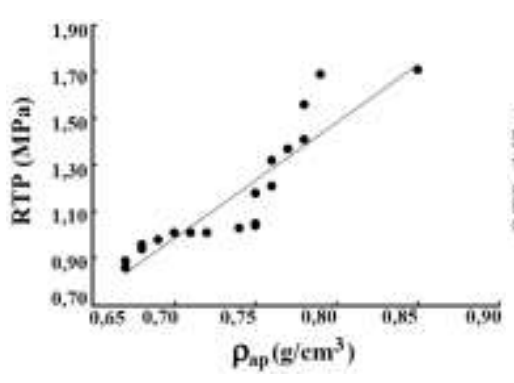

(a)

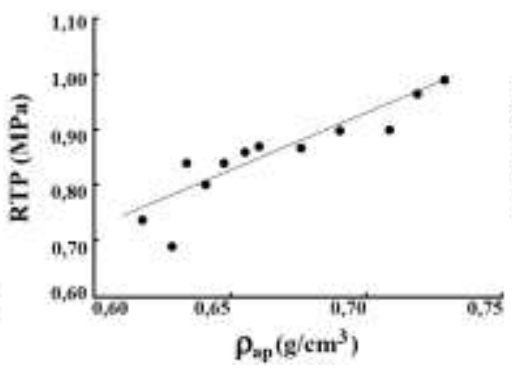

(b)

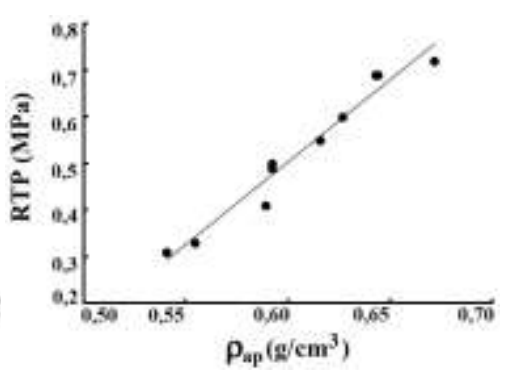

(c) 
Tabela 8 - Resultados das estatísticas da regressão e da ANOVA

\begin{tabular}{|c|c|c|c|c|c|}
\hline \multicolumn{6}{|c|}{ PU-MAMONA } \\
\hline Estatísticas da Regressão & $\begin{array}{c}\text { R Múltiplo } \\
0,91\end{array}$ & $\begin{array}{c}\mathbf{R}^{\mathbf{2}} \\
0,82 \\
\end{array}$ & $\begin{array}{c}\mathbf{R}^{2} \text { Ajustado } \\
0,81\end{array}$ & $\begin{array}{c}\text { Erro Padrão } \\
0,11\end{array}$ & $\begin{array}{c}\text { Obs. } \\
20\end{array}$ \\
\hline ANOVA & GL & SQ & MQ & $\mathbf{F}$ & F de Significação \\
\hline Regressão & 1 & 1,043 & 1,043 & 82,195 & $3,949 \mathrm{E}-08$ \\
\hline Erro Residual & 18 & 0,228 & 0,013 & & \\
\hline Total & 19 & 1,271 & & & \\
\hline Equação & \multicolumn{5}{|c|}{$\mathrm{RTP}=4,973 \cdot \rho_{\mathrm{ap}}-2,497$} \\
\hline \multicolumn{6}{|c|}{ UREIA-FORMALDEÍDO } \\
\hline Estatísticas da Regressão & $\begin{array}{c}\text { R Múltiplo } \\
0,84 \\
\end{array}$ & $\begin{array}{c}\mathbf{R}^{\mathbf{2}} \\
0,78 \\
\end{array}$ & $\begin{array}{c}\mathbf{R}^{2} \text { Ajustado } \\
0,74\end{array}$ & $\begin{array}{c}\text { Erro Padrão } \\
0,02\end{array}$ & $\begin{array}{c}\text { Obs. } \\
12\end{array}$ \\
\hline ANOVA & $\mathbf{G L}$ & SQ & MQ & $\mathbf{F}$ & F de Significação \\
\hline Regressão & 1 & 0,056 & 0,056 & 13,34 & 0,0016 \\
\hline Erro Residual & 10 & 0,015 & 0,004 & & \\
\hline Total & 11 & 0,071 & & & \\
\hline Equação & \multicolumn{5}{|c|}{$\mathrm{RTP}=1,381 \cdot \rho_{\mathrm{ap}}-0,729$} \\
\hline & \multicolumn{4}{|c|}{ PURBOND } & \\
\hline Estatísticas da Regressão & $\begin{array}{c}\text { R Múltiplo } \\
0,92\end{array}$ & $\begin{array}{c}\mathbf{R}^{\mathbf{2}} \\
0,87\end{array}$ & $\begin{array}{c}\mathbf{R}^{\mathbf{2}} \text { Ajustado } \\
0,83\end{array}$ & $\begin{array}{c}\text { Erro Padrão } \\
0,05\end{array}$ & $\begin{array}{c}\text { Obs. } \\
12\end{array}$ \\
\hline ANOVA & GL & SQ & MQ & $\mathbf{F}$ & F de Significação \\
\hline Regressão & 1 & 0,210 & 0,210 & 58,03 & $5,761 \mathrm{E}-06$ \\
\hline Erro Residual & 10 & 0,005 & 0,003 & & \\
\hline Total & 11 & 0,215 & & & \\
\hline Equação & \multicolumn{5}{|c|}{$\mathrm{RTP}=0,934 \cdot \rho_{\mathrm{ap}}-0,166$} \\
\hline
\end{tabular}

\section{Conclusões}

Pode-se concluir que:

(a) os materiais elaborados com resina ureiaformaldeído e Purbond apresentaram os melhores resultados para o módulo de elasticidade na flexão;

(b) os compósitos fabricados apenas com ureiaformaldeído exibiram os maiores valores do módulo de ruptura na flexão;

(c) os painéis elaborados com a resina Purbond apresentaram os melhores resultados para a resistência à tração perpendicular; e

(d) pelos resultados obtidos da análise de variância, constatou-se ser possível estimar a resistência à tração perpendicular dos materiais com o conhecimento da densidade aparente para os três tipos de resinas utilizadas, com a característica comum do aumento da resistência pelo aumento na densidade.

Em linhas gerais, as propriedades físicas e mecânicas investigadas foram compatíveis com os limites estipulados pelos documentos normativos de referência, e o coeficiente de variação dos materiais fabricados apresentou-se próximo dos obtidos em outras pesquisas na mesma temática, evidenciando a boa homogeneidade obtida na fabricação dos compósitos.

Em pesquisas futuras, espera-se trabalhar com outras proporções entre partículas com as mesmas ou com outras espécies de madeira, e com outras frações de adesivo, objetivando a busca por materiais com melhores propriedades físicas que os aqui investigados.

\section{Referências}

ALBUQUERQUE, C. E. C. Interações de Variáveis no Ciclo de Prensagem de Aglomerados. Curitiba, 2002. 150 f. Tese (Doutorado em Ciências Florestais) - Setor de Ciências Agrárias, Universidade Federal do Paraná, Curitiba, 2002.

AMERICAN NATIONAL STANDARD. ANSI A208.1: particleboard. Gaithersburg, 1999.

\section{AMERICAN SOCIETY FOR TESTING AND}

MATERIALS. ASTM D-1037: standard methods of evaluating properties of wood-base fiber and particles materials. Philadelphia, 2006. 
ASSOCIAÇÃO BRASILEIRA DE NORMAS TÉCNICAS. NBR 14810: chapas de madeira aglomerada: parte 2: requisitos: parte 3: métodos de ensaio. Rio de Janeiro, 2006.

BERALDO, A. L.; JOSÉ, F. J. Painéis Prensados de Partículas de Bambu e Adesivo Poliuretana à Base de Óleo de Mamona. In: ENCONTRO BRASILEIRO EM MADEIRAS E EM ESTRUTURAS DE MADEIRA, 10., São Pedro, SP, 2006. Anais... São Pedro, SP, 2006.

BLANCHET, P.; CLOUTIER, A.; RIEDL, B. Particleboard Made From Hammer Milled Black Spruce Bark Residues. Wood Science and Technology, v. 34, n. 1, p. 11-19, Mar. 2000. CAMPOS, R. A. et al. Análise da Qualidade de Chapas de Madeira Aglomerada com Casca de Pinhão Manso. In: ENCONTRO BRASILEIRO EM MADEIRAS E EM ESTRUTURAS DE MADEIRA, 13., Vitória, 2012. Anais... Vitória, 2012.

COMMERCIAL STANDARD. CS 236-66: mat formed wood particleboard. 1968.

DIAS, F. M. et al. Relation Between the Compaction Rate and Physical and Mechanical Properties of Particleboards. Materials Research, v. 8, n. 3, p. 329-333, 2005.

\section{EUROPEAN STANDARD. EN 312:}

particleboards: specifications. Bruxelas, 2003.

GUIMARÃES JÚNIOR, J. B. et al. Avaliação das Propriedades Físicas de Painéis Aglomerados de Embaúba. In: ENCONTRO BRASILEIRO EM

MADEIRAS E EM ESTRUTURAS DE MADEIRA, 13, Vitória, 2012. Anais... Vitória, 2012.

INSTITUTO BRASILEIRO DE GEOGRAFIA E ESTATÍSTICA. Anuário eEtatístico do Brasil. Rio de Janeiro, 2010. Disponível em:

http://www.ibge.gov.br/home/estatistica/indicador es/agropecuaria/lspa/lspa_201002_5.shtm>.

Acesso em: 17 out. 2011.

IWAKIRI, S. et al. Produção de Painéis de Madeira Aglomerada de Cinco Espécies de Pínus Tropicais. Floresta e Ambiente, v. 8, n. 1, p. 137 142, jan./dez. 2001.

LIMA, M. D. F. Utilização de Resíduos das Espécies Dipterix polyphylla (Cumarurana), Dipterix odorata (Cumaru) e Brosimun paranarioides (Amapá) na Produção de Painéis de Madeira Aglomerada Com Resina Poliuretana à Base de Óleo de Mamona. Amazonas, 2012. 152 f. Dissertação (Mestrado em Engenharia Civil) - Escola de Engenharia, Universidade Federal do Amazonas, Amazonas, 2012.
MALONEY, T. M. Modern Particleboard and Dry-Process Fiberboard Manufacturing. 2nd. São Francisco: Freeman, 1993.

MATTOS, R. L. G; GONÇALVES, R. M.; CHAGAS, F. B. Painéis de Madeira no Brasil: panorama e perspectivas. BNDES Setorial, Rio de Janeiro, n. 27, p. 121-156, mar. 2008.

MENDES, R. F. et al. Avaliação de Espécies de Rápido Crescimento Para a Produção de Painéis Glomerados. In: ENCONTRO BRASILEIRO EM MADEIRAS E EM ESTRUTURAS DE MADEIRA, 13, Vitória, 2012. Anais... Vitória, ES, 2012.

MENDES, R. F. et al. Painéis Aglomerados Produzidos Com Bagaço de Cana em Associação Com Madeira de Eucalipto. Scientia Forestalis, Piracicaba, v. 38, n. 86, p. 285-295, jun. 2010.

NASCIMENTO, M. F. Chapas de Partículas Homogêneas: madeiras do Nordeste do Brasil. São Carlos, 2003. Tese (Doutorado em Engenharia de Materiais) - Interunidades em Ciências e Engenharia de Materiais, Universidade de São Paulo, São Carlos, 2003.

SILVA, S. A. M.; LAHR, F. A. R. Chapas de Partículas Confeccionadas Com Resíduos de Madeiras Tropicais de Baixa Densidade. In: RECICLAGEM de Resíduos Para a Construção Civil. Belo Horizonte: Editora da Universidade Federal de Ouro Preto, 2008. cap. 14.

SILVA, S. A. M. et al. Particleboard Manufactured with Bicomponent Polyurethane Resin base on Castor Oil. International Journal of Composite Materials, v. 2, n. 6, p. 115-118, 2012.

TOMASELLI, I. Tendências de Mudanças na Indústria de Painéis. Revista da Madeira, Caxias do Sul, p. 36-40, 2000.

TRIANOSKI, R. Avaliação do Potencial de Espécies Florestais 2010 Crescimento, Para Produção de Painéis de Madeira Aglomerada. Curitiba, 2010. 260 f. Dissertação (Mestrado em Engenharia Florestal) - Escola de Engenharia, Universidade Federal do Paraná, Curitiba, 2010. 


\section{Wilson Henrique Negrão}

Departamento de Engenharia Civil, Faculdade de Engenharia de Itha Solteira | Universidade Estadual Paulista Júlio de Mesquita Filho | Alameda Bahia, 550, Centro | Itha Solteira - SP - Brasil | CEP 15385-000 | Tel.: (18) 3743-1266 | E-mail: negrao@terra.com.br

\section{Sérgio Augusto Mello da Silva}

Departamento de Engenharia Civil, Faculdade de Engenharia de Itha Solteira | Universidade Estadual Paulista Júlio de Mesquita Filho | E-mail: sams@dec.feis.unesp.br

\section{André Luis Christoforo}

Departemento de Engenharia Civil | Universidade Federal de São Carlos | Rodovia Washington Luís, km 235 | São Carlos - SP - Brasil | CEP 36307-352 | Tel.: (16) 3351-8262 | E-mail: alchristoforo@yahoo.com.br

\section{Francisco Antonio Rocco Lahr}

Escola de Engenharia de São Carlos | Universidade de São Paulo | Av. Trabalhador Sãocarlense, 400, Centro | São Carlos - SP - Brasil | CEP 13566-590 | Tel.: (016) 3373-8206 | E-mail: frocco@sc.usp.br

\section{Revista Ambiente Construído}

Associação Nacional de Tecnologia do Ambiente Construído

Av. Osvaldo Aranha, $99-3^{\circ}$ andar, Centro

Porto Alegre - RS - Brasil

CEP 90035-190

Telefone: +55 (51) 3308-4084

Fax: +55 (51) 3308-4054

www.seer.ufrgs.br/ambienteconstruido

E-mail: ambienteconstruido@ufrgs.br 